\title{
Clinical and Microbiological Profile of Patients with Bloodstream Infections Caused by Burkholderia cepacia Complex
}

\author{
Tasneem Siddiqui $^{1}$ Chinmoy Sahu ${ }^{1}$ Sangram Singh Patel ${ }^{1}$ Ujjala Ghoshal ${ }^{1}$ \\ ${ }^{1}$ Department of Microbiology, Sanjay Gandhi Postgraduate Institute \\ of Medical Sciences, Lucknow, Uttar Pradesh, India \\ Address for correspondence Chinmoy Sahu, MD, DNB, Department \\ of Microbiology, Sanjay Gandhi Postgraduate Institute of Medical \\ Sciences, Rae Bareli Road, Lucknow, 226014, Uttar Pradesh, India \\ J Lab Physicians 2022;14:312-316. \\ (e-mail: sahu.chinmoy@gmail.com).
}

\begin{abstract}
Keywords

- Burkholderia cepacia complex

- bloodstream infections

- antibiotic sensitivity
\end{abstract}

Introduction Burkholderia cepacia complex (BCC) is an emerging pathogen causing nosocomial bloodstream infections (BSIs), and its treatment is challenging due to its multidrug resistance. In India, there is a dearth of data on BSIs caused by BCC, therefore, an updated study is required to know the clinical and microbiological profile of these patients. We aimed to study the clinical epidemiology and antibiotic susceptibility pattern of BCC isolated from blood samples in our hospital.

Materials and Methods This observational study was conducted from January 2019 to December 2020 at a tertiary care center in northern India. All the blood cultures were done on an automated blood culture system. All BCC isolates of BSI were identified depending on their morphological properties and biochemical reactions, and underwent the matrix-assisted laser desorption ionization time-of-flight mass spectrometry system to confirm diagnosis. Antibiotic susceptibility testing was done as per Clinical Laboratory and Standards Institute guidelines.

Results BCC was isolated from $30 \mathrm{BSI}$ patients over a 2-year period. Sixty-six percent (20/30) of patients had cancer and a majority of them were undergoing chemotherapy. The most common predisposing factors were the use of steroids, immunosuppressive drugs, and chemotherapy (93.3\%), central lines (83.3\%), use of higher antibiotics (60\%), and diabetes mellitus type $2(60 \%)$. The most common species isolated were $B$. cepacia (64\%) and B. cenocepacia (30\%). Isolates showed highest sensitivity to minocycline (100\%), ceftazidime $(73.3 \%)$, and meropenem $(70 \%)$ and the least to ticarcillinclavulanate.

Conclusion BCC is an emerging pathogen causing BSIs, especially in malignancy patients. Minocycline can be a good choice for these bacteria. published online

February 9, 2022
DOI https://doi.org/ $10.1055 / \mathrm{s}-0042-1742634$ ISSN 0974-2727. (c) 2022. The Indian Association of Laboratory Physicians. All rights reserved.

This is an open access article published by Thieme under the terms of the Creative Commons Attribution-NonDerivative-NonCommercial-License, permitting copying and reproduction so long as the original work is given appropriate credit. Contents may not be used for commercial purposes, or adapted, remixed, transformed or built upon. (https://creativecommons.org/ licenses/by-nc-nd/4.0/)

Thieme Medical and Scientific Publishers Pvt. Ltd., A-12, 2nd Floor, Sector 2, Noida-201301 UP, India 


\section{Introduction}

Burkholderia cepacia complex (BCC) is ubiquitous in nature, present in water, soil, and plants. BCC is comprised of gramnegative non-lactose-fermenting bacteria. Burkholderia cepacia has emerged as an opportunistic nosocomial pathogen since the 1980s, particularly in patients with debilitating diseases. Based on molecular analysis, Pseudomonas cepacia has been separated from Pseudomonas and renamed as $B$. cepaci. BCC is a cluster of at least ten closely related genomic species, which includes B. cepacia, $B$. multivorans, $B$. cenocepacia, and others that can be differentiated by molecular and biochemical methods. ${ }^{1}$ It can cause fatal necrotizing pneumonia and bacteremia, especially in patients with cystic fibrosis or chronic granulomatous diseases. ${ }^{2-4}$ Pneumonia, meningitis, urinary tract infections, and bloodstream infections (BSIs) are caused by BCC in noncystic fibrosis patients. $\mathrm{BCC}$ is a new pathogen that is causing significant morbidity and mortality in hospitalized patients, owing to its high intrinsic antibiotic resistance. ${ }^{5}$ It has always been a tedious task for a routine microbiological laboratory to identify the nonfermenting gram-negative bacilli (NFGNBs), and poor laboratory proficiency in the identification of BCC prevails worldwide, including our own country. For this reason, reports of disease due to this organism are rare in India. ${ }^{6-10}$ Differentiation of BCC from $P$. aeruginosa is important as BCC is intrinsically resistant to aminoglycosides, firstand second-generation cephalosporins, and traditional antipseudomonal penicillins. Worldwide, among pathogenic NFGNB, BCC is the fourth most common after $P$. aeruginosa, Acinetobacter calcoaceticus-baumannii complex, and Stenotrophomonas maltophilia. ${ }^{5}$ In India, there is a dearth of data on BSIs caused by BCC; therefore, in this observational study, we aimed to advance our understanding of BSIs due to BCC, by analyzing the clinical epidemiology and antibiotic susceptibility pattern of these BCC isolates in our patient population.

\section{Materials and Methods}

\section{Study Design and Selection of Cases}

This is an observational study conducted from January 2019 till December 2020 in microbiology laboratory of a tertiary care center of Northern India. All consecutive, nonduplicate isolates of BCC from BSIs considered clinically significant were included in the study.

\section{Sample Processing and Identification of Isolates}

All the blood cultures were done on automated blood culture system (Becton Dickinson Diagnostics, BD Headquarters, Franklin Lakes, New Jersey, USA). Nonlactose fermenting colonies on MacConkey agar that were motile, catalase and oxidase positive, and slender gram-negative bacilli with bipolar staining were subjected to biochemical reactions like Indole production, citrate utilization, nitrate reduction, urea hydrolysis, oxidation fermentation test (Hugh and Leifson medium), decarboxylase test, and esculin hydrolysis. After confirming as BCC, tests for genomic speciation, growth at $42^{\circ} \mathrm{C}$, ONPG test, pigment production, $10 \%$ lactose utilization, were also performed. Motility is tested with hanging-drop preparation since the semisolid agar medium for detecting motility of fermentative organisms may not be suitable for this NFGNB. ${ }^{11}$ Phenazine pigments (red, maroon, yellow) are produced by BCC that impart distinctive color to the colonies, which are helpful in making identification. ${ }^{11}$ These isolates were confirmed by MALDI-TOF-MS using the Biotyper system according to manufacturer recommendations (VITEK MS, bioMérieux, United States).

\section{Antimicrobial Susceptibility Testing}

Antibiotic susceptibility testing was done using conventional (Kirby Bauer disc diffusion method on Müller Hinton agar) and automated method (Vitek 2) ${ }^{12}$ selecting antibiotics recommended for testing against BCC by the Clinical Laboratory and Standards Institute $(\mathrm{CLSI})^{13}$ : ceftazidime, trimethoprim sulfamethoxazole, meropenem, ticarcillin-clavulanate, levofloxacin, piperacillin-tazobactam, and minocycline. Antibiotic sensitivity was interpreted as per CLSI, 2019. ${ }^{13}$

A study proforma was created, which included patient demographic data, clinical data, and predisposing factors such as (1) presence of neutropenia, (2) central lines, (3) parenteral nutrition, (4) blood products, (5) intensive care unit stay, (6) intubation/ventilation, (7) immunosuppressive drugs use, (8) chemotherapy, (9) prior use of higher antibiotics, and (10) dialysis.

\section{Statistical Analysis}

Statistical Package of Social Sciences, version-23 (SPSS-23, IBM, Chicago, Illinois, United States) were used for descriptive statistics. Categorical data were described using numbers and percentages.

\section{Results}

BCC was isolated from 30 BSI patients over a 2-year period. The age of the patient population ranged from 5 to 84 years, with a mean age of 38 (standard deviation: 11.5 ) years. The number of males and females enrolled in the study was 21 and 9, respectively, with a M:F ratio of 2.3:1. The incidence of nosocomial B. cepacia infections was very low in our hospital (0.32 per 1,000 admissions). The majority of patients in the study had hematological malignancy $(17 / 30 ; 56 \%)$ and were mostly on chemotherapy. Other patients had coronary artery disease $(5 / 30 ; 17 \%)$, solid organ malignancy $(3 / 30 ; 10 \%)$, endstage renal disease requiring dialysis $(2 / 30 ; 7 \%)$, pancreatitis $(2 / 30 ; 7 \%)$, and acute encephalitis $(1 / 30 ; 3 \%)$.

The majority of Burkholderia complex species involved in clinical infections of the study were B. cepacia (19/30,64\%) and B. cenocepacia (9/30,30\%), but there was one isolate each of $B$. vietnamiensis and $B$. contaminans. The most common predisposing factors present in the study population were use of steroids, immunosuppressive drugs, and chemotherapy (93.3\%) (-Table 1). Out of 30 patients, five patients suffered from coronavirus disease 2019 (COVID-19) pneumonia and had had bacterial coinfection with BCC. The identifications of 
Table 1 Predisposing factors present in patient population of the study

\begin{tabular}{|l|l|}
\hline Predisposing factors & $\begin{array}{l}\text { Number of } \\
\text { patients }(\boldsymbol{n}, \%)\end{array}$ \\
\hline Neutropenia & $12(40 \%)$ \\
\hline Central lines & $25(83.3 \%)$ \\
\hline $\begin{array}{l}\text { Steroids/immunosuppressive } \\
\text { drugs/chemotherapy }\end{array}$ & $28(93.3 \%)$ \\
\hline Diabetes & $18(60 \%)$ \\
\hline Parenteral nutrition & $5(16.7 \%)$ \\
\hline Higher antibiotics used before & $18(60 \%)$ \\
\hline Blood products & $14(46.7 \%)$ \\
\hline Intubation/mechanical ventilation & $6(20 \%)$ \\
\hline Dialysis & $7(23.3 \%)$ \\
\hline
\end{tabular}

these isolates were B. cepacia, B. cenocepacia, and B. vietnamiensis recovered from 3,1 , and 1 patients, respectively.

All 30 isolates showed $100 \%$ sensitivity to minocycline. Ceftazidime and meropenem were found to be sensitive in $73.3 \%$ and $70 \%$, respectively, of the isolates. However, the sensitivities of levofloxacin, trimethoprim sulfamethoxazole, and piperacillin-tazobactam were $66.7,60$, and $53.3 \%$, respectively. Only $16.7 \%$ of isolates were sensitive to ticarcillin-clavulanate (-Fig. 1).

\section{Discussion}

The majority of data on nosocomial BCC infections in the literature are limited to epidemics, ${ }^{4}$ whereas our data show no epidemics and only sporadic cases of BCC infections. Ku et al studied BCC bacteremia and found BCC infection to be commoner in male patients $(66.7 \%){ }^{14}$ Similarly our study also had a male preponderance with $70 \%$ of infected patients being male. The most common species of Burkholderia complex isolated in the study were $B$. cepacia and $B$. cenocepacia, which is similar to other studies. $^{15,16}$ The most frequent risk factors in the study population were use of steroids, immunosuppressive drugs, and chemotherapy, which could be because most of the patients in the study had malignancy, which itself is an immunocompromised state. Besides, these patients received chemotherapy as well as lots of immunosuppressive drugs that cause low immunity in the patients. Hence, they were vulnerable to opportunistic infections such as Burkholderia, which is itself nonharmful to healthy people. Five patients suffered from COVID-19 pneumonia and had had bacterial coinfection with BCC. Long-term use of steroids also causes an increase in the rate of infections due to deranged cellular immunity, which could be cause of such infections in both cancer and COVID-19-positive patients. Other risk factors revealed in these patients were invasive procedures, mostly central line insertions like chemo ports as well as peripherally inserted central catheters. As central lines are essential in malignancy patients for long-term chemotherapy infusions, infection of central lines might happen during the insertion procedure as well as during the maintenance period. Biofilm formation in the central lines might cause bacteria to harbor and cause infections in the central lines associated with BSIs. Blood products through central lines might help in the formation of biofilms that might cause infections in central lines in these cases. Neutropenia will be caused by

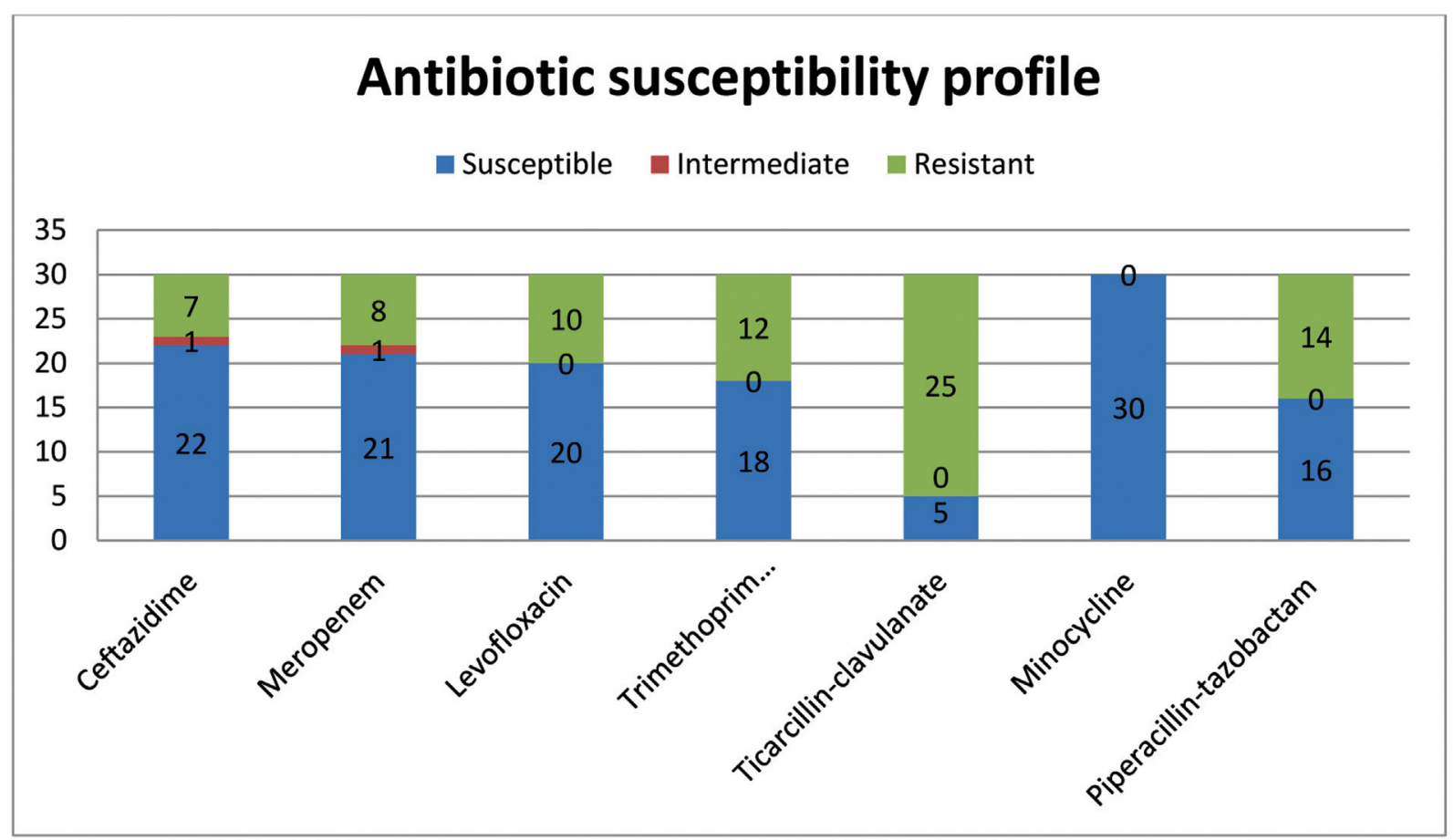

Fig. 1 Results of antimicrobial susceptibility testing of isolates of Burkholderia cepacia complex causing bloodstream infection. 
chemotherapy due to myelosuppression during the treatment. Diabetes itself causes an infection due to the deranged blood sugar level in patients. All the above predisposing factors that have been reported in earlier studies ${ }^{14,17}$ were also found to be present in our cases that might have resulted in Burkholderia infections.

BCC is intrinsically resistant to many antibiotics. It has been well documented that it has intrinsic resistance to aminoglycosides, first- and second-generation cephalosporins, and traditional antipseudomonal penicillins. The multidrug resistance of $B$. cepacia has been credited to an impermeable selective outer membrane, an efflux pump mechanism, and/or the production of an inducible chromosomal $\beta$-lactamase. ${ }^{18-20}$ In the current study, antibiotic susceptibility tests revealed that minocycline was the most effective antibiotics against BCC with susceptibility percentage (100\%), followed by ceftazidime (73.3\%), meropenem (70\%), levofloxacin (66.7\%), and trimethoprim sulfamethoxazole (60\%). They exhibited moderate sensitivity to piperacillin-tazobactam (53.3\%), while BCC isolates showed high resistance to ticarcillin-clavulanic acid (83.3\%). The study done by Kady et al reported B. cepacia isolates to be $100 \%$ susceptible to meropenem, ceftazidime, and piperacillin-tazobactam, followed by cefepime (87.5\%), cotrimoxazole, and minocycline (50\%). All strains (100\%) were resistant to both ciprofloxacin and ticarcillin-clavulanate. ${ }^{21}$ Omar et al in their study found that $B$. cepacia isolates were $88.5 \%$ susceptible to meropenem and $60 \%$ to ceftazidime. All are $100 \%$ resistant to both co-trimoxazole and ciprofloxacin. ${ }^{22}$

Comparing the results of this study most of the isolates were sensitive to minocycline meropenem and ceftazidime as suggested by the CLSI guidelines, with varying sensitivity to other antimicrobials tested. On the other hand, this study showed high resistance (83.3\%) to ticarcillin-clavulanic acid, which was almost similar to Kady et al (100\%). From various studies finally, we could conclude that there were variations in the results of drug susceptibility that may be because of various antibiotic policies followed in different hospitals. So, there is need to properly isolate and do antibiotic sensitivity testing for each strain for better patient management.

\section{Conclusion}

As BCC is intrinsically resistant to many antibiotics, laboratory diagnosis with identification of these bacteria becomes mandatory. Moreover, due to the lack of advanced diagnostic facilities to confirm the etiological agent, they are reported as NFGNB or simply as Pseudomonas species. Thus, to facilitate diagnosis and appropriate antibiotic treatment, all NFGNB grown in clinical specimens should be speciated. Our study highlights BCC as an emerging pathogen causing BSIs, especially in malignancy patients. Minocycline can be a good choice for these bacteria.

\section{Conflict of Interest}

None declared.

\section{References}

1 Mahenthiralingam E, Vandamme P, Campbell ME, et al. Infection with BCC genomovars in patients with cystic fibrosis: virulent transmissible strains of genomovar III can replace Bukholderia multivorans. Clin Infect Dis 2001;33:1469-1475

2 Siddiqui AH, Mulligan ME, Mahenthiralingam E, et al. An episodic outbreak of genetically related Burkholderia cepacia among noncystic fibrosis patients at a university hospital. Infect Control Hosp Epidemiol 2001;22(07):419-422

3 Estivariz CF, Bhatti LI, Pati R, et al. An outbreak of Burkholderia cepacia associated with contamination of albuterol and nasal spray. Chest 2006;130(05):1346-1353

4 Nasser RM, Rahi AC, Haddad MF, Daoud Z, Irani-Hakime N, Almawi WY. Outbreak of Burkholderia cepacia bacteremia traced to contaminated hospital water used for dilution of an alcohol skin antiseptic. Infect Control Hosp Epidemiol 2004;25(03): 231-239

5 LiPuma JJ CB, Lum GD, Vandamme PAR. Burkholderia, Stenotrophomonas, Ralstonia, Cupriavidus, Pandoraea, Brevundimonas, Comamonas and Acidovorax. In: Murray PR BE, Jorgensen JH, Landry ML, Pfaller MA, eds. Manual of Clinical Microbiology. Washington, DC: ASM Press; 2007:749-769

6 Gautam V, Arora A, Madhup SK, et al. Burkholderia cepacia complex in septicaemic non-cystic fibrosis cases from two tertiary care hospitals in north India. Indian J Med Res 2010; 131:829-832

7 Gautam V, Ray P, Das A, et al. Two cases of Burkholderia cenocepacia in septicemic patients. Jpn J Infect Dis 2008;61(02): 133-134

8 Gautam V, Ray P, Puri GD, et al. Investigation of Burkholderia cepacia complex in septicaemic patients in a tertiary care hospital, India. Nepal Med Coll J 2009;11(04):222-224

9 Gautam V, Ray P, Vandamme P, et al. Identification of lysine positive non-fermenting gram negative bacilli (Stenotrophomonas maltophilia and Burkholderia cepacia complex). Indian J Med Microbiol 2009;27(02):128-133

10 Mukhopadhyay C, Bhargava A, Ayyagari A. Two novel clinical presentations of Burkholderia cepacia infection. J Clin Microbiol 2004;42(08):3904-3905

11 Winn W Jr, Allen S, Janda W, Koneman E, Procop G, Schreckenberger $P$, et al. Nonfermenting gram negative bacilli. In: Koneman's Color Atlas and Textbook of Diagnostic Microbiology. 6th edition. USA: Lippincott Williams and Wilkins Company; 2006: 305-391

12 Ling TKW, Tam PC, Liu ZK, Cheng AFB. Evaluation of VITEK 2 rapid identification and susceptibility testing system against gramnegative clinical isolates. J Clin Microbiol 2001;39(08): 2964-2966

13 Clinical and Laboratory Standards Institute (CLSI) Performance Standards for Antimicrobial Susceptibility Testing. CLSI Supplement M-100 29th edition. Wayne, PA: CLSI; 2019

$14 \mathrm{Ku}$ NS, Han SH, Kim CO, et al. Risk factors for mortality in patients with Burkholderia cepacia complex bacteraemia. Scand J Infect Dis 2011;43(10):792-797

15 Mahenthiralingam E, Urban TA, Goldberg JB. The multifarious, multireplicon Burkholderia cepacia complex. Nat Rev Microbiol 2005;3(02):144-156

16 Reik R, Spilker T, Lipuma JJ. Distribution of Burkholderia cepacia complex species among isolates recovered from persons with or without cystic fibrosis. J Clin Microbiol 2005;43 (06):2926-2928

17 Bressler AM, Kaye KS, LiPuma JJ, et al. Risk factors for Burkholderia cepacia complex bacteremia among intensive care unit patients without cystic fibrosis: a case-control study. Infect Control Hosp Epidemiol 2007;28(08):951-958

18 Burns JL, Wadsworth CD, Barry JJ, Goodall CP. Nucleotide sequence analysis of a gene from Burkholderia (Pseudomonas) 
316 Bloodstream Infections by BCC Siddiqui et al.

cepacia encoding an outer membrane lipoprotein involved in multiple antibiotic resistance. Antimicrob Agents Chemother 1996;40(02):307-313

19 Aronoff SC. Outer membrane permeability in Pseudomonas cepacia: diminished porin content in a beta-lactam-resistant mutant and in resistant cystic fibrosis isolates. Antimicrob Agents Chemother 1988;32(11):1636-1639

20 Trépanier S, Prince A, Huletsky A. Characterization of the penA and penR genes of Burkholderia cepacia 249 which encode the chromosomal class A penicillinase and its LysR-type transcrip- tional regulator. Antimicrob Agents Chemother 1997;41(11): 2399-2405

21 Kady HE, Mohamed ONE, Abaza AF, Zidan YHM. Burkholderia cepacia complex among intensive care unit patients in two private hospitals in Alexandria. Inter J Sci Technol Research 2018;7(01):102-109

22 Omar N, El Raouf HA, Okasha H, Nabil N. Microbiological assessment of Burkholderia cepacia complex (BCC) isolates in Alexandria Main University Hospital. Alex J Med 2015;51(01): $41-46$ 